\title{
Improved methods for haemozoin quantification in tissues yield organ-and parasite-specific information in malaria-infected mice
}

\author{
Katrien Deroost ${ }^{1}$, Natacha Lays ${ }^{1}$, Sam Noppen ${ }^{2,3}$, Erik Martens ${ }^{1}$, Ghislain Opdenakker ${ }^{1}$
} and Philippe E Van den Steen ${ }^{1 *}$

\begin{abstract}
Background: Despite intensive research, malaria remains a major health concern for non-immune residents and travelers in malaria-endemic regions. Efficient adjunctive therapies against life-threatening complications such as severe malarial anaemia, encephalopathy, placental malaria or respiratory problems are still lacking. Therefore, new insights into the pathogenesis of severe malaria are imperative. Haemozoin $(\mathrm{Hz})$ or malaria pigment is produced during intra-erythrocytic parasite replication, released in the circulation after schizont rupture and accumulates inside multiple organs. Many in vitro and ex vivo immunomodulating effects are described for $\mathrm{Hz}$ but in vivo data are limited. This study aimed to improve methods for $\mathrm{Hz}$ quantification in tissues and to investigate the accumulation of $\mathrm{Hz}$ in different organs from mice infected with Plasmodium parasites with a varying degree of virulence.
\end{abstract}

Methods: An improved method for extraction of $\mathrm{Hz}$ from tissues was elaborated and coupled to an optimized, quantitative, microtiter plate-based luminescence assay with a high sensitivity. In addition, a technique for measuring $\mathrm{Hz}$ by semi-quantitative densitometry, applicable on transmitted light images, was developed. The methods were applied to measure $\mathrm{Hz}$ in various organs of C57BL/6 J mice infected with Plasmodium berghei ANKA, P. berghei NK65 or Plasmodium chabaudi AS. The used statistical methods were the Mann-Whitney $U$ test and Pearsons correlation analysis.

Results: Most $\mathrm{Hz}$ was detected in livers and spleens, lower levels in lungs and kidneys, whereas sub-nanomolar amounts were observed in brains and hearts from infected mice, irrespectively of the parasite strain used. Furthermore, total $\mathrm{Hz}$ contents correlated with peripheral parasitaemia and were significantly higher in mice with a lethal $P$. berghei ANKA or $P$. berghei NK65-infection than in mice with a self-resolving $P$. chabaudi AS-infection, despite similar peripheral parasitaemia levels.

Conclusions: The developed techniques were useful to quantify $\mathrm{Hz}$ in different organs with a high reproducibility and sensitivity. An organ-specific Hz deposition pattern was found and was independent of the parasite strain used. Highest $\mathrm{Hz}$ levels were identified in mice infected with lethal parasite strains suggesting that $\mathrm{Hz}$ accumulation in tissues is associated with malaria-related mortality.

Keywords: Chemo-luminescence, Densitometry, Haemozoin quantification, Malaria pigment, Plasmodium

\footnotetext{
* Correspondence: Philippe.VandenSteen@rega.kuleuven.be

'Laboratory of Immunobiology, Rega Institute, University of Leuven, Leuven, Belgium

Full list of author information is available at the end of the article
}

\section{Biomed Central}

(c) 2012 Deroost et al.; licensee BioMed Central Ltd. This is an Open Access article distributed under the terms of the Creative Commons Attribution License (http://creativecommons.org/licenses/by/2.0), which permits unrestricted use, distribution, and reproduction in any medium, provided the original work is properly cited. 


\section{Background}

With growing world populations in tropical countries, every year more people become exposed to malaria. As many areas of endemic malaria transmission overlap with regions of poverty, direct and indirect burdens of this infectious disease are important. In particular, a small percentage of the malaria-infected patients develop life-threatening complications such as severe malarial anaemia, encephalopathy, placental malaria or respiratory problems, even if they have similar peripheral parasitaemia levels compared to patients with mild or asymptomatic malaria [1]. Efficient adjunct therapies against these immunopathological complications are still not available. Therefore, studying the mechanisms of disease development in severe malaria is paramount.

Inside the red blood cell (RBC), almost $80 \%$ of the haemoglobin $(\mathrm{Hb})$ is degraded, which means that high amounts of toxic haem (which is rapidly oxidized to haemin) are liberated in the food vacuole of the parasite capable of generating reactive oxygen species and damaging cell membranes and proteins $[2,3]$. As a detoxification mechanism, the parasite biocrystallizes the haemin molecules into insoluble haemozoin $(\mathrm{Hz})$ crystals or malaria pigment $[4,5]$. When the schizont ruptures, $\mathrm{Hz}$ is released into the circulation and is rapidly removed by phagocytes inside several organs. Multiple in vitro and ex vivo pro-inflammatory and immunosuppressive effects of $\mathrm{Hz}$ have been described (reviewed in [4-7]). However, few in vivo data about the effects of $\mathrm{Hz}$ on the immune system exist. As large amounts of $\mathrm{Hz}$ are produced during infection and accumulate inside multiple organs, $\mathrm{Hz}$ may be important for the progress towards malaria-associated pathologies. This hypothesis is further strengthened by the fact that abundant $\mathrm{Hz}$ has been observed in brains [8-10] and placentas [11-13] from malaria patients with cerebral and placental complications, respectively. In addition, $\mathrm{Hz}$ was detected in brains of mice with cerebral symptoms $[14,15]$ and in lungs of mice with malaria-associated acute respiratory distress syndrome (MA-ARDS) [16].

Experimental mouse models offer useful tools to study malaria-related disease mechanisms. Depending on the mouse-parasite combination, different aspects of human malaria can be mimicked and investigated, even if these models are not exact replicas and should thus be extrapolated with caution. In this study, C57BL/6 J mice were infected with three different parasite strains with a varying degree of pathogenicity. The Plasmodium berghei ANKA (PbANKA) parasite induces typical symptoms of cerebral malaria (CM), such as paralysis or coma and mice succumb within seven to nine days. In this mouse model, the pathology critically depends on activation of leukocytes, including $\mathrm{CD}^{+} \mathrm{T}$ cells, and a local inflammatory reaction [1]. Although sequestration of Plasmodium falciparum in the brain is strongly associated with CM in patients, it is unclear whether specific cyto-adherence of PbANKA occurs in the brain [17]. However, local parasite accumulation in the brain is thought to be an important feature of this model [18]. Mice infected with Plasmodium berghei NK65 (PbNK65) do not develop such an encephalopathy but rather die from severe respiratory problems between nine and eleven days post-infection [16]. This respiratory pathology closely resembles human MA-ARDS, as in both mice and patients leukocytes (predominantly macrophages and lymphocytes) and infected RBCs (iRBCs) accumulate in the lungs, resulting in the disruption of endothelial barriers, severe edema and intra-alveolar hyaline membrane formation [16,19,20]. Plasmodium chabaudi AS (PcAS)-infections are self-limiting and the mice are able to recover. Moreover, C57BL/6 mice mount a protective immune response against PcAS-parasites mediated by phagocytes, $\mathrm{CD}^{+}{ }^{+} \mathrm{T}$ cells and specific antibodies, which is very similar to the immune response generated against P. falciparum in humans [21].

To investigate the organ-specific $\mathrm{Hz}$ deposition in these three mouse models, novel techniques are described in the present study to accurately quantify the $\mathrm{Hz}$ content in tissues. These methods were implemented to compare the amount of $\mathrm{Hz}$ between various organs and between similar organs from mice infected with parasites of different pathogenicity. Most $\mathrm{Hz}$ was found in livers and spleens. Far less $\mathrm{Hz}$ was detected in lungs and kidneys, whereas limited amounts of $\mathrm{Hz}$ were observed in hearts and brains, irrespectively of the parasite species. In addition, more $\mathrm{Hz}$ was found in mice infected with PbNK65 or PbANKA compared with PcAS-infected mice despite of similar peripheral parasitaemia levels.

\section{Methods}

\section{Chemical products}

All chemicals were purchased from Sigma-Aldrich (Bornem, Belgium), unless otherwise stated.

\section{Mice and parasites}

C57BL/6 J mice (seven to nine weeks old) were obtained from Janvier (Le Genest-Saint-Isle, France) and placed in a conventional animal house with food and water ad libitum. Parasite growth in mice was supported by supplementing the drinking water with $0.375 \mathrm{mg} / \mathrm{mL}$ 4-amino benzoic acid. Mice were intraperitoneally infected with $10^{4}$ iRBCs by serial passage of tail vein blood obtained from a mouse that had been infected with one of the following parasite strains: PcAS, P $b$ NK65 (kind gifts of the late Prof. D Walliker, University of Edinburgh, Scotland, UK) or PbANKA (Cl15CY1, a kind gift of Prof. C Jansse, Leiden University Medical Centre, The Netherlands). The percentage of infected erythrocytes in the peripheral blood was determined by 
microscopic analysis after Giemsa staining. Mice were sacrificed at the indicated time points after infection and blood was removed by heart puncture. Mice were perfused with Dulbecco's phosphate-buffered saline (PBS) (Lonza, Verviers, Belgium) to remove circulating iRBCs from the organs. Livers, spleens, kidneys, lungs, hearts and brains were removed, weighed and stored at $-80^{\circ} \mathrm{C}$ until further analysis. A part of the liver, spleen, lung and kidney was embedded in Tissue-Tek O.C.T. Compound (Sakura Finetek Europe B.V., Alphen aan den Rijn, The Netherlands) and frozen in liquid nitrogen-cooled isopentane for histological analysis. All experiments were approved by the local ethical committee (License LA121251, Belgium).

\section{Haemozoin quantification in organ cryosections by densitometric analysis}

Cryosections with a thickness of $7 \mu \mathrm{m}$ were prepared from frozen livers, spleens, lungs and kidneys and imaged by light microscopy. Transmitted light images were taken through a 20x/0.8 Plan-Apochromat objective of an Axiovert $200 \mathrm{M}$ microscope equipped with an AxioCam MRm camera (Zeiss, Göttingen, Germany). For $\mathrm{Hz}$ quantification on liver sections, images were obtained from two rows of three consecutive fields. The densitometric analysis was performed with the AxioVision 4.6 software with a home-written script and the relative quantity of $\mathrm{Hz} / \mu \mathrm{m}^{2}$ was calculated with the formula as shown in Figure 1G. The densitometric value (DV) of a pixel reflects the intensity of transmitted light at this position in the section. The densitometric background (DB) was determined in each picture by calculating the mean DV of all pixels with a DV above an empirically determined threshold. To test the linearity of the densitometric method to measure $\mathrm{Hz}$ on cryosections, gelatin blocks containing different concentrations of synthetic $\mathrm{Hz}(\mathrm{sHz})$ were analysed. $\mathrm{sHz}$ was prepared as described previously [22] and was homogenized and added in different concentrations to a $10 \%$ gelatin-PBS solution at $37^{\circ} \mathrm{C}$ in a 24-well plate. Upon solidification on ice (to prevent sedimentation of $\mathrm{sHz}$ ), $\mathrm{sHz}$ containing gelatin blocks were cut out of the wells, embedded in O.C.T and frozen at $-80^{\circ} \mathrm{C}$. For each concentration, five to six images were analysed from one section/sHz block and this was done for two blocks.

\section{Haem quantification by colorimetric analysis and haem- enhanced luminescence}

The Fe-ions in the haemin molecules that constitute the $\mathrm{Hz}$ crystal are in the oxidized state $\left(\mathrm{Fe}^{3+}\right)$. Therefore, a dilution series of a haematin stock solution $(10 \mu \mathrm{M}-$ $1.2 \mathrm{nM}$ ), prepared by dissolving haemin in $100 \mathrm{mM}$ $\mathrm{NaOH}, 2 \%$ SDS and $3 \mathrm{mM}$ EDTA, was used as a standard to compare methods. In an alkaline environment, haematin produces a brown colour measurable spectrophotometrically (Biotech Powerwave XS) at $405 \mathrm{~nm}$ [23]. Background absorbance was evaluated from a blank sample and subtracted from the measurements. The method for quantification by luminescence was based on the method of Schwarzer et al. [24] and was optimized for working in a 96-well plate and modified according to Yuan et al. [25]. Different concentrations of haematin were added in 96-well plates suitable for luminescence (Perkin Elmer, Waltham, MA, USA) and diluted in a solution containing $\mathrm{NaOH}$ and $\mathrm{Na}_{2} \mathrm{CO}_{3}$ (four volumes of $100 \mathrm{mM} \mathrm{NaOH}, 2 \%$ SDS and $3 \mathrm{mM}$ EDTA and one volume of $1 \mathrm{M} \mathrm{Na}_{2} \mathrm{CO}_{3}, \mathrm{pH}$ 10.4) (final volume $50 \mu \mathrm{L})$. After addition of $100 \mu \mathrm{L}$ luminol $(100 \mu \mathrm{g} / \mathrm{mL}$ 3 -aminophtalhydrazide) and $100 \mu \mathrm{L}$ of peroxide (7\% tertbutyl hydroperoxide), both dissolved in the $\mathrm{NaOH} /$ $\mathrm{Na}_{2} \mathrm{CO}_{3}$-solution, light emitted in the presence of $\mathrm{Fe}^{3+}$ (present in the haematin core) was measured during one second using a Thermo Luminoskan Ascent apparatus. Peroxide catalysis into oxygen by $\mathrm{Fe}^{3+}$ is a fast reaction. Therefore, special care was taken to keep the time between the addition of the peroxide and the luminescence measurements minimal and as similar as possible between the different wells (maximum time deviation between individual wells was eight seconds). A sigmoidal relationship between the haematin concentration and the luminescence (events/sec) was obtained. Background luminescence was evaluated from a blank sample and subtracted from the measurements. The above method for haem-enhanced luminescence was used for all measurements unless differently stated. The time-dependence of the luminescence signal was measured with $125 \mathrm{nM}$ haematin in duplicate every ten seconds for eight minutes during a kinetic reading without any other samples in the plate to allow fast repetitive reading of these two wells. A lag-time of twelve seconds existed between the addition of the peroxide and the start of the measurement, which was partly attributable to a shaking step.

\section{Haemozoin determination in tissues and trophozoites}

To extract haemozoin from perfused mouse tissues, approximately 30-60 mg (liver, spleen, kidney or lung), half brain or a full heart were homogenized with the Precellys Lysing Kit (VWR, Leuven, Belgium) in minimum five volumes of a solution containing $50 \mathrm{mM}$ Tris/ $\mathrm{HCl} \mathrm{pH} \mathrm{8.0,} 5 \mathrm{mM} \mathrm{CaCl}_{2}, 50 \mathrm{mM} \mathrm{NaCl}$ and $1 \%$ Triton $\mathrm{X}-100$. The homogenate was supplemented with $1 \%$ Proteinase $\mathrm{K}$ and incubated overnight at $37^{\circ} \mathrm{C}$. The next day the proteinase $\mathrm{K}$ digest was sonicated (VialTweeter, Hielscher Ultrasonics GmbH, Teltow, Germany) for $1 \mathrm{~min}(10 \mathrm{~W}$, pulse $0.5 \mathrm{sec})$ and centrifuged at $11,000 \mathrm{x} \mathrm{g}$ for $45 \mathrm{~min}$. The supernatant was discarded and the pellet was washed three times in $100 \mathrm{mM} \mathrm{NaHCO}, \mathrm{pH} 9.0$ and $2 \%$ SDS with subsequent sonication and centrifugation for 
$30 \mathrm{~min}$ to remove degraded tissue, free haem and $\mathrm{Hb}$. After the third wash, the pellet $(\mathrm{Hz})$ was dissolved and sonicated in $100 \mathrm{mM} \mathrm{NaOH}, 2 \%$ SDS and $3 \mathrm{mM}$ EDTA to form haematin and centrifuged to pellet any remaining insoluble material. To confirm that the isolated material was indeed $\mathrm{Hz}$, it was examined for its birefringence character. For this purpose, isolated $\mathrm{Hz}$ that was washed three times as described above, was subsequently washed in distilled $\mathrm{H}_{2} \mathrm{O}$ to remove the salts, smeared on a glass slide and monitored by polarized light microscopy with a 40x/1.3 oil EC Plan-Neofluar objective of an Axiovert $200 \mathrm{M}$ microscope.

To isolate $\mathrm{Hz}$ from trophozoites, heparinized blood was obtained by heart puncture from $P b N K 65$-infected mice and trophozoites were cultivated ex vivo overnight and harvested as described [26]. After determination of the total red cell number and the percentage of iRBCs, $\mathrm{Hz}$ was extracted from the cells as described above but without proteinase $\mathrm{K}$ treatment and subsequently dissolved as described.

The extracted $\mathrm{Hz}$ was measured in different dilutions with the above-mentioned protocol for haem quantification by luminescence. A dilution series of haematin $(10 \mu \mathrm{M}-1.2 \mathrm{nM})$ was used as a standard. The unknown $\mathrm{Hz}$ concentration was calculated from the calibration curve of the haematin concentration (nM) versus luminescence (events/sec). Background luminescence was evaluated from a blank sample and subtracted from the measurements. The amount of $\mathrm{Hz}$ (fmol or $\mathrm{pmol}$ haematin/mg tissue) was multiplied with the total weight of the concerning organ and expressed as pmol or nmol haematin/organ. An accuracy limit was estimated for each organ separately.

\section{Statistical analysis}

$P$-values for the differences between two groups were calculated with the Mann-Whitney $U$-test, using the GraphPad Prism software (GraphPad Software, San Diego, CA, USA). The same software was used for linear regression analyses and for calculating Spearman correlation coefficients. The slopes of the individual regression lines of the different groups were compared online [27]. A $p$-value less than $0.05(p<0.05)$ was taken as statistically significant.

\section{Results}

\section{Haemozoin detection by densitometric analysis}

Pigment distribution was investigated on unstained cryosections from various organs of mice infected with PbNK65 or PcAS and monitored with light microscopy. $\mathrm{Hz}$ was observed on transmitted light images from infected mouse organs as brown pigments and was absent in organ sections from uninfected mice as is shown for the liver (Figure 1A-F). The pigment was found equally distributed throughout the liver (Figure 1B-C), whereas in the spleen (Figure 1D), the lungs (Figure 1E) or the kidneys (Figure 1F), it was located in the red pulp, the interstitial tissue or clustered in presumably the glomeruli, respectively. Brains and hearts contained such low amounts of $\mathrm{Hz}$ that it was almost unnoticeable on unstained sections. To estimate the amount of $\mathrm{Hz}$ on organ cryosections, semi-quantitative densitometry with the AxioVision 4.6 software using a home-written script was applied. The linearity of this densitometric method was investigated on cryosections from gelatin-blocks with different concentrations of $\mathrm{sHz}$. A broad linear relationship was found between the $\mathrm{sHz}$ concentration and the obtained relative densitometric value (Figure 1G). The detected $\mathrm{Hz}$ signal was converted into the relative quantity of $\mathrm{Hz} / \mu^{2} \mathrm{~m}^{2}$ tissue as calculated with the formula described in Figure 1G. This technique was used to measure $\mathrm{Hz}$ in liver cryosections from non-infected, $P c A S$ and PbNK65-infected mice. Despite similar mean peripheral parasitaemia levels in both groups of mice (PcAS 18\%; PbNK65 12.7\%; $p=0.4$ ), significantly more $\mathrm{Hz} / \mu \mathrm{m}^{2}$ tissue was detected in livers of $\mathrm{PbNK} 65$-infected mice compared with PcAS-infected mice (Figure $1 \mathrm{H}$ ). This technique combines in-situ information with relative quantification and can be used to measure $\mathrm{Hz}$ in organs with a high and evenly distributed $\mathrm{Hz}$ content such as livers. However, this method is poorly applicable for organs in which the pigment is not equally distributed, e.g. spleen, lungs and kidneys. In brains and hearts, the amounts of $\mathrm{Hz}$ were too low to be quantified by densitometry.

\section{Comparison of haem quantification by different techniques}

Because the densitometric analysis is not suitable to quantify $\mathrm{Hz}$ in tissue sections from all organs, a more sensitive technique was designed to determine $\mathrm{Hz}$ in organ extracts. Several methods for quantifying $\mathrm{Hz}$ in blood are described in literature, including a colorimetric [23] and a chemo-luminescence assay [24]. These two techniques were adapted to a 96-well plate format and the sensitivity was compared by measuring different concentrations of haematin produced when haemin or $\mathrm{Hz}$ is dissolved in an alkaline environment. A sigmoidal relationship was obtained between the haematin concentration and the blank-subtracted absorbance at $405 \mathrm{~nm}$ (Figure 2A) or the blank-subtracted luminescence with the reagent concentrations used as described for a cuvette-based system by Schwarzer et al. [24] (Figure 2B). In the 96-well plate format adapted for measuring absorbance or luminescence with a plate reader, both techniques detected the presence of haematin starting from a minimal concentration of $1 \mu \mathrm{M}$. To improve the sensitivity, the chemo-luminescent assay was adjusted by stabilizing the $\mathrm{pH}$ around 10.4 and by 


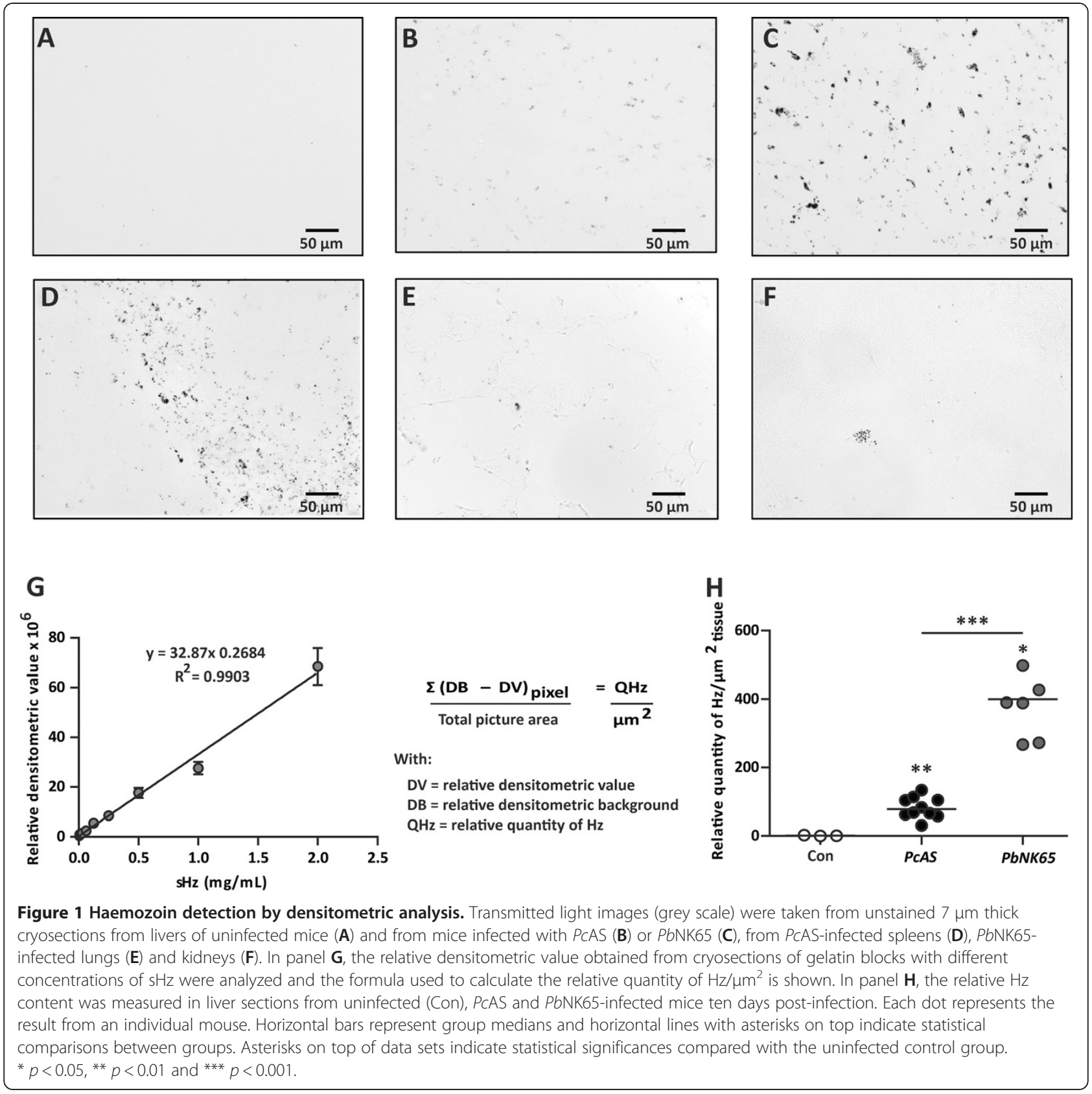

raising the concentrations of luminol and peroxide 100fold. In this way, a detection limit around $100 \mathrm{nM}$ was obtained (Figure $2 \mathrm{C}$ ). Furthermore, the time-dependence of the luminescence signal was examined by measuring the emitted light of a single concentration during a kinetic measurement. With the described protocol for haem-enhanced luminescence in a 96-well plate format, emitted light was measured when the luminescence signal was almost maximal (Figure 2D). The sensitivity of this 96-well plate-based assay is significantly lower than the sensitivity of the cuvette-based method described by Schwarzer et al. [24]. However, the current 96-well based method has the advantage of a higher throughput and the sensitivity appeared sufficient to measure $\mathrm{Hz}$ in organ extracts. To extract $\mathrm{Hz}$ from organs, the method described by Sullivan et al. [15] was optimized. The most important modification was the addition of an overnight proteinase $\mathrm{K}$ digestion step that eliminated high background signals e.g. in lung samples. After several wash steps to remove any free haemin, the $\mathrm{Hz}$ crystals were converted to free haematin by dissolving in a strong alkaline environment, so that the haematin concentration could be measured. Since the extraction procedure involved washing steps in the presence of SDS, possible 


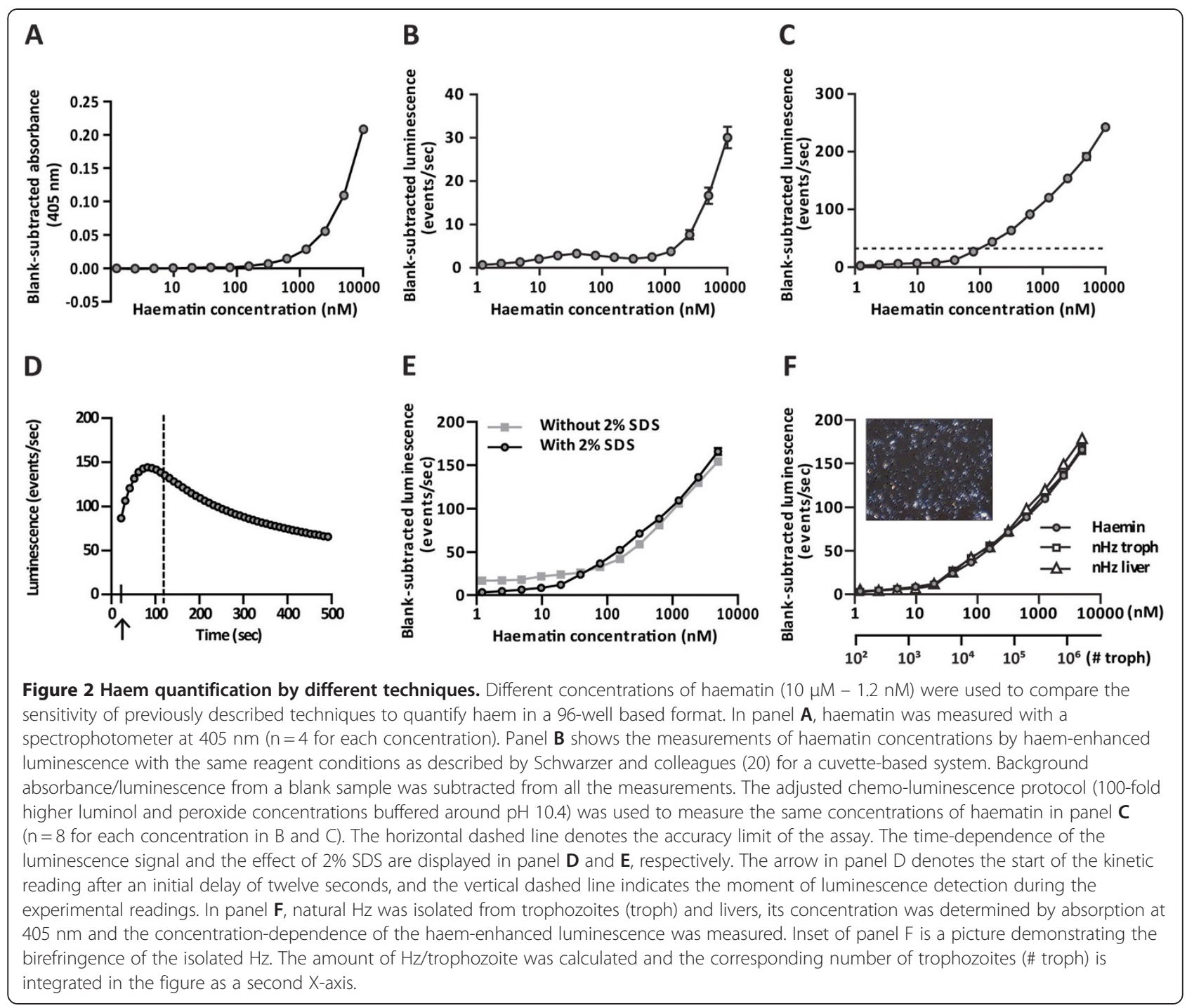

quenching of the emitted light by SDS was investigated and had no effect on the luminescence catalyzed by haematin- $\mathrm{Fe}^{3+}$ (Figure 2E).

As a final check up for the appropriateness of the assay for quantifying natural $\mathrm{Hz}$, the concentration dependence of the haem-enhanced luminescence measured with the plate reader was studied with natural $\mathrm{Hz}$ purified from trophozoites and from the liver. As shown in Figure 2F, haemin and $\mathrm{Hz}$ derived from trophozoites or livers behaved similarly in the assay confirming the suitability of using haemin as a standard for deducing the $\mathrm{Hz}$ concentration in the samples. In addition, as birefringence is a typical feature of $\mathrm{Hz}$ [28], $\mathrm{Hz}$ isolated from the liver was spread on a glass slide and monitored by polarized light microscopy. This confirmed that the isolated material consisted mainly of $\mathrm{Hz}$ (Figure 2F, inset).

\section{Quantitative analysis of haemozoin in various organs of} mice infected with different parasite species

The optimized haem-enhanced luminescence technique was used to study differences in the amount of $\mathrm{Hz}$ between various organs and between the same organs of mice infected with parasites of different pathogenicity (PbANKA, PbNK65 or PcAS). Mice were sacrificed at the indicated times post-infection and perfused systemically. Even though no difference was found in the amount of $\mathrm{Hz}$ before and after perfusion (Additional file 1), it seemed more reasonable to apply perfusion on all samples tested. In this way, there could be no doubt that the detected $\mathrm{Hz}$ represented organ-trapped $\mathrm{Hz}$ and not $\mathrm{Hz}$ present in the circulation. Quantification of the total amount of $\mathrm{Hz}$ per organ revealed that most $\mathrm{Hz}$ was present in livers followed by spleens (Figure $3 \mathrm{~A}-\mathrm{B}$ ). Far less $\mathrm{Hz}$ was detected in lungs and kidneys (Figure $3 \mathrm{C}-\mathrm{D}$ ), whereas subnanomolar 

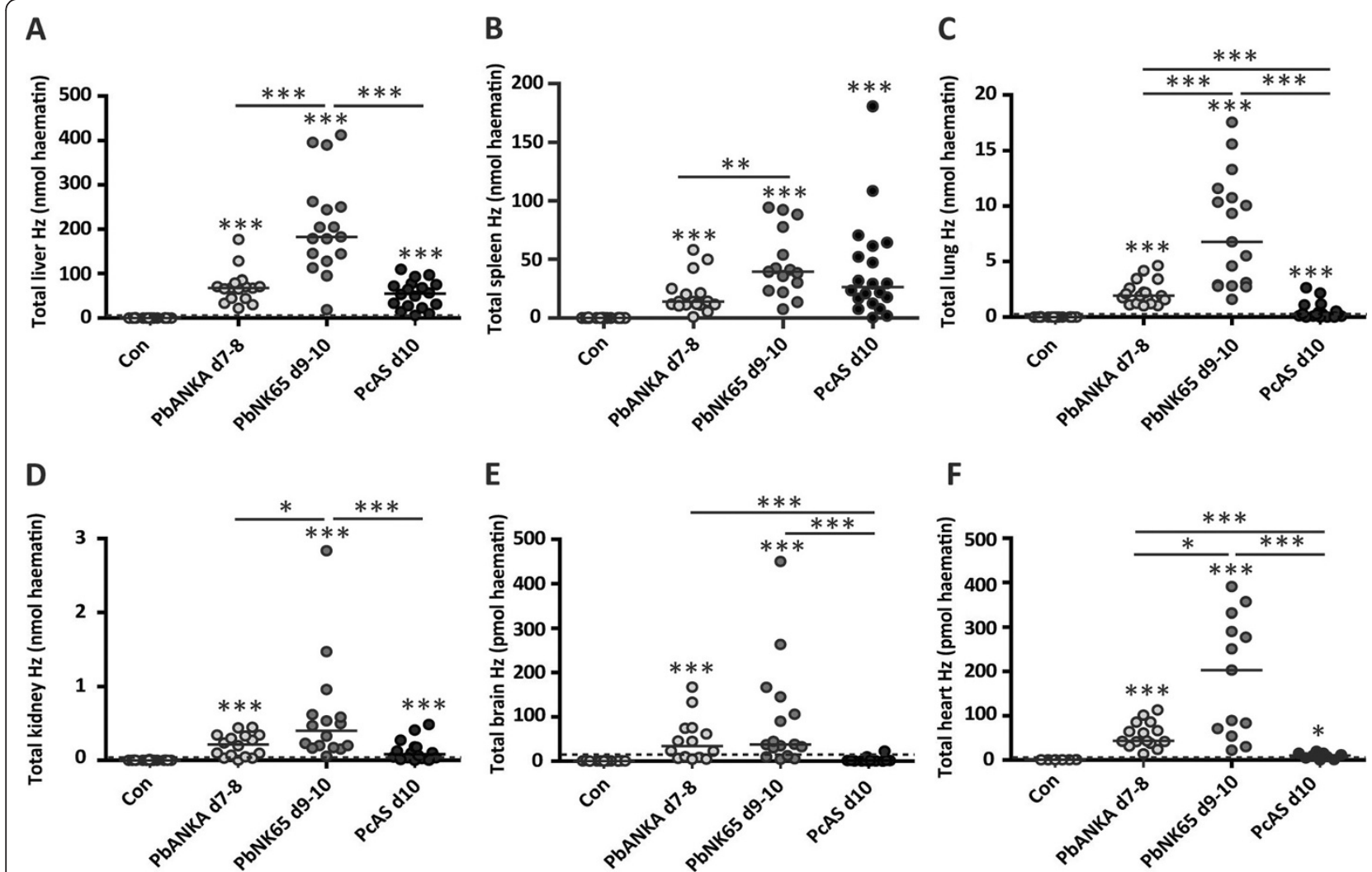

Figure 3 Quantification of haemozoin in tissues. C57BL/6 J mice were infected with $10^{4}$ PbANKA, PbNK65 or PcAS parasites or were left uninfected (Con). At the indicated time intervals after infection, mice were dissected after heart puncture and perfusion. Extracted $\mathrm{Hz}$ from 30 - $60 \mathrm{mg}$ tissue of livers $(\mathbf{A})$, spleens $(\mathbf{B})$, lungs $(\mathbf{C})$ and kidneys $(\mathbf{D})$, from half brains $(\mathbf{E})$ and from whole hearts $(\mathbf{F})$ were quantified by haem-enhanced luminescence and expressed as nmol haematin/organ (liver, spleen, lungs and kidneys) or pmol haematin/organ (brain and heart). Each group consisted of 15 to 20 mice, with each dot indicating individual data points. Horizontal dashed lines were used to denote the accuracy limit of the assay for each organ separately. Horizontal bars represent group medians and horizontal lines with asterisks on top indicate statistical comparisons between groups. Asterisks on top of data sets indicate statistical significances compared with the uninfected control group. ${ }^{*} p<0.05,{ }^{* *} p<0.01$ and ${ }^{* * *} p<0.001$.

levels of $\mathrm{Hz}$ were found in brains and hearts, irrespectively of the parasite species used (Figure 3E-F). Livers, lungs, kidneys and hearts from $P b N K 65$-infected mice nine to ten days post-infection contained significantly more $\mathrm{Hz}$ compared with the same organs from $P b A N K A$-infected mice seven to eight days post-infection or PcAS-infected mice ten days post-infection, even though livers of PcASinfected mice were significantly larger $(p<0.0001$ for liver weights between $P c$ AS d10 and PbNK65 d9-10 and between PcAS d10 and PbANKA d7-8). In addition, lungs and hearts from PbANKA-infected mice seven to eight days post-infection had significantly more $\mathrm{Hz}$ compared with the same organs from PcAS-infected mice after ten days of infection. The total amount of $\mathrm{Hz}$ was similar in spleens of $P c A S$ and $P b N K 65$-infected mice ten days post-infection (Figure 3B), although the amount of $\mathrm{Hz} / \mathrm{mg}$ spleen tissue was six-fold lower in mice infected with PcAS compared to PbNK65 (median value was 1164.4 pmol haematin/mg spleen for $P b N K 65$ and 199.9 pmol haematin/mg spleen for PcAS; $p<0.0001)$. This was compensated by the three- to four-fold larger spleen size in PcAS-infected mice $(p<0.0001)$. Furthermore, similar amounts of $\mathrm{Hz}$ were observed in brains from $P b$ NK65 and PbANKA-infected mice, whereas less $\mathrm{Hz}$ was found in brains of PcAS-infected mice.

\section{Different total haemozoin levels in PcAS-infected mice and Plasmodium berghei-infected mice}

As brains and hearts contained only subnanomolar amounts of $\mathrm{Hz}$, individual total $\mathrm{Hz}$ levels were calculated by making the sum of organ-specific $\mathrm{Hz}$ levels from liver, spleen, lungs and kidneys. Mice infected with parasites of the $P$. berghei strains contained significantly more $\mathrm{Hz}$ than mice infected with PcAS-parasites (Figure 4A). Moreover, the total amounts of $\mathrm{Hz}$ correlated with the peripheral parasitaemia levels for all parasite strains (Figure 4B and C). PbANKA and PbNK65-parasites had a similar Hzproduction pattern, i.e. comparable amounts of $\mathrm{Hz}$ at similar peripheral parasitaemia levels. In contrast, PcASinfected mice seemed to have significantly less $\mathrm{Hz}$ in 


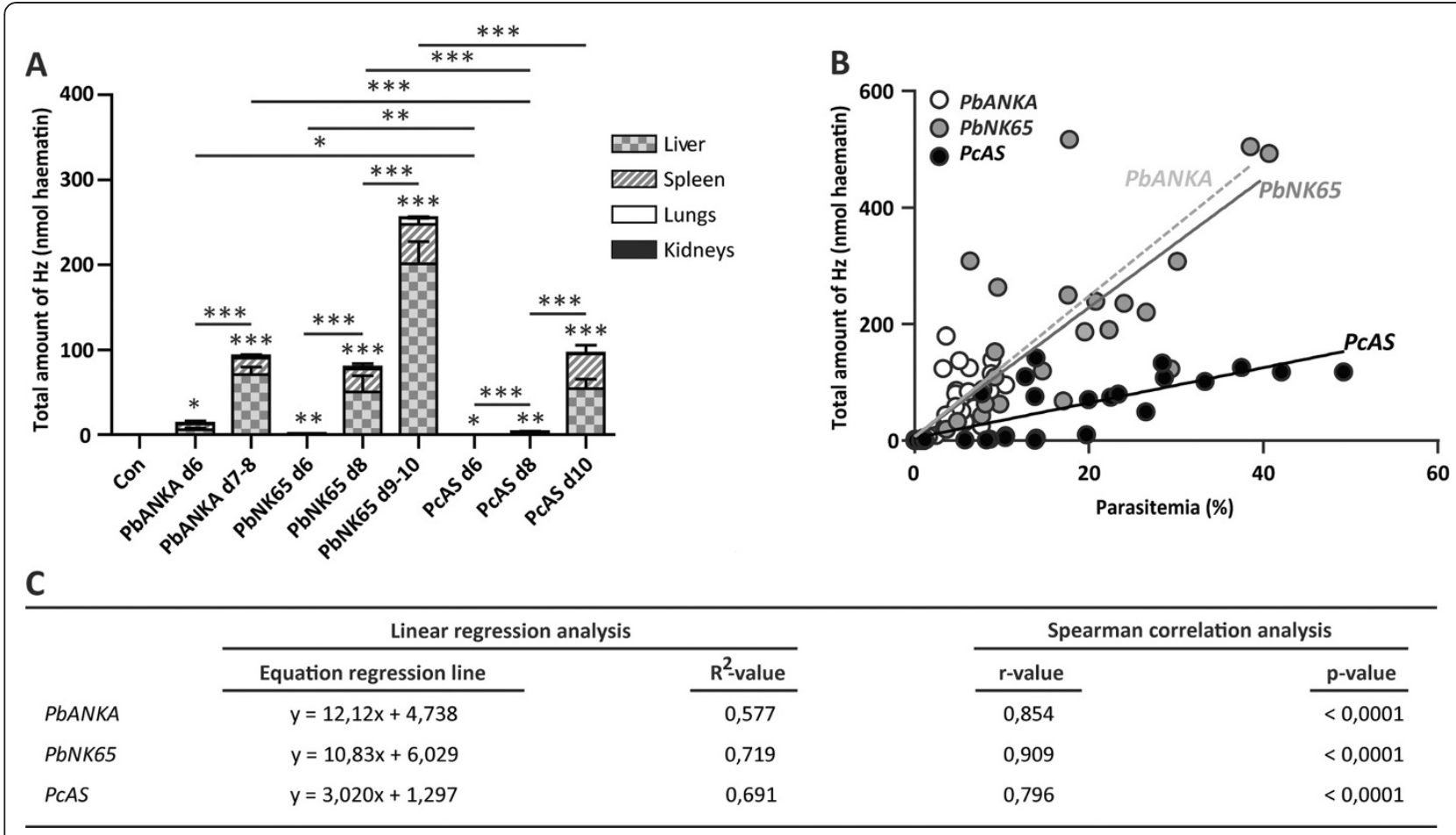

Figure 4 PcAS-infected mice contain less total $\mathrm{Hz}$ than Plasmodium berghei-infected mice. The total amount of Hz (nmol haematin)/mouse was estimated from the sum of the $\mathrm{Hz}$ content in the individual organs. Panel $\mathbf{A}$ shows the average amount of $\mathrm{Hz}$ found in $\mathrm{C} 57 \mathrm{BL} / 6 \mathrm{~J}$ mice infected with PbANKA, PbNK65 or PCAS on different time points post-infection, with the contribution of each organ to the total amount of $\mathrm{Hz} \pm \mathrm{SEM}$. Asterisks on top of data sets indicate the statistical significances compared with the uninfected control group. Horizontal lines with asterisks on top indicate statistical differences between infected groups. ${ }^{*} p<0.05,{ }^{* *} p<0.01$ and ${ }^{* * *} p<0.001$. In panel $\mathbf{B}$, the total amount of $\mathrm{Hz}$ (nmol haematin) was correlated with the level of parasitaemia (\%) for each parasite strain separately and the individual regression lines are shown. The regression line of PbANKA was prolonged under the form of a hatched line to better distinguish it from the PbNK65 line. The equation of the regression lines and the $\mathbf{R}^{2}$-values, together with Spearman $r$-values and $p$-values are shown in panel $\mathbf{C}$.

relation with their parasitaemia compared with $\mathrm{PbANKA}$ or $\mathrm{PbNK} 65$ as indicated by the lower slope on the regression curve. The difference between the slopes of the regression lines was significantly different between $\mathrm{PbNK} 65$ and PcAS-infected mice $(p<0.0001)$.

\section{Discussion}

The first observations of black pigment in necroptic spleens and brains go back to the $18^{\text {th }}$ Century (reviewed in [5]). About 130 years later, a publication mentioned brown-grey colourations of brain, spleen and liver, which turned out to arise from pigment deposition. At first believed to be melatonin, it was later linked to a parasitic disease. Presently, many in vitro and ex vivo immunomodulating effects have been ascribed to $\mathrm{Hz}$ [4-7]. However, data about the fate and properties of $\mathrm{Hz}$ in the in vivo situation are still scarce. $\mathrm{Hz}$ is released in the circulation in considerable amounts after schizont rupture where it may interact with a whole range of different cell types. The majority of the liberated $\mathrm{Hz}$ is presumably captured and phagocytosed by circulating and tissue resident monocytes/ macrophages in which it can persist for a long time. In this way, $\mathrm{Hz}$ may be capable of causing considerable inflammation that might progress to tissue injury. In this study, techniques for sensitively quantifying the amount of $\mathrm{Hz}$ in tissues were examined and the organ-specific $\mathrm{Hz}$ content was compared between parasite species with a varying degree of pathogenicity.

As $\mathrm{Hz}$ crystals were observed on unstained cryosections from livers, spleens, lungs and kidneys, a technique for estimating the amount of $\mathrm{Hz}$ in these sections by densitometric analysis was developed. As $\mathrm{Hz}$ is proportionally distributed throughout the liver, the estimation of the amount of $\mathrm{Hz}$ by densitometry was quite reliable. In other organs, however, $\mathrm{Hz}$ was found in specific structures such as the red pulp in the spleen, the interstitial tissue in the lungs or presumably the glomeruli in the kidneys. This may in part be attributed to the differential localization of tissue-resident phagocytes. This implies that $\mathrm{Hz}$ distribution is a confounding factor for the accuracy of the $\mathrm{Hz}$ measurements on organ cryosections by densitometry. In addition, this technique is time-consuming, labour-intensive, semi-quantitative and not suitable for organs with a low $\mathrm{Hz}$ content and was thus not further 
explored. Therefore, a more sensitive, analytical and quantitative method for determining the $\mathrm{Hz}$ content in tissues was investigated. To isolate $\mathrm{Hz}$ from organs, a protocol described by Sullivan and colleagues [15] was modified. The main adaptation was the digestion of the homogenates with proteinase $\mathrm{K}$. This digestion eliminated high background signals, which were presumably due to the binding of $\mathrm{Hb}$ to otherwise insoluble extracellular matrix components. Upon conversion of the isolated $\mathrm{Hz}$ into soluble haematin, a chemo-luminescence assay was used for quantification. This assay was based on the method of Schwarzer et al. [24] and adapted to microtiter plate format. The obtained sensitivity with the optimized protocol was lower compared with the haem-enhanced luminescence assay described by Schwarzer et al. This was not due to quenching of the luminescence signal by SDS nor was it caused by the altered time frame during which the emitted light was measured (two seconds/sample versus approximately ninety six seconds/plate), but probably originated from the use of different luminescence detector systems (cuvette system versus microplate reader). Nevertheless, the microplate-adjusted approach offers the advantage of measuring several samples in varying concentrations simultaneously with a sensitivity that is optimal for the quantification of $\mathrm{Hz}$ in malaria-infected organs.

As an application, the distribution of $\mathrm{Hz}$ throughout the body of infected mice was studied and compared between diverse parasite strains with varying pathogenicity. Sullivan and colleagues already quantified the $\mathrm{Hz}$ content in brains, livers and spleens of mice $[15,29]$ and in human placentas [11], but no detailed comparison between organs and between parasite species was described. Almost 95\% of the total pool of $\mathrm{Hz}$ was found in livers and spleens. This was expected as large volumes of blood are filtered through these organs and both contain a vast population of tissue-resident monocytes/macrophages capable of rapidly removing the crystalline material from the circulation by means of phagocytosis. It was also important to consider the liver and spleen sizes when determining the total $\mathrm{Hz}$ amounts, as these sizes evolve in a different way during infection with different parasites (i.e. induction of hepatosplenomegaly by PcAS). As the absolute $\mathrm{Hz}$ concentration in the organs could be determined by the luminescence assay, this was easily taken into account by multiplication with the organ weights.

Furthermore, substantial amounts of $\mathrm{Hz}$ were detected in lungs of malaria-infected mice. In a new mouse model of MA-ARDS [16], considerable amounts of $\mathrm{Hz}$ were observed on histological sections of the lungs. By quantifying the $\mathrm{Hz}$ content in the lungs, significantly higher $\mathrm{Hz}$ levels were validated in lungs from $P$. berghei-infected mice (lung pathology) compared to PcAS-infected mice (no lung pathology), indicating that $\mathrm{Hz}$ may have a role in the development of malaria-associated lung disease.

Low but detectable amounts of $\mathrm{Hz}$ were found in kidneys, hearts and brains of malaria-infected mice. Most $\mathrm{Hz}$ was detected in kidneys and hearts from $P b N K 65$-infected mice ten days post-infection compared with $P b A N K A$ and $P c$ AS-infected mice seven to eight and ten days postinfection, respectively. However, a different pattern was observed in the brains, i.e. $\mathrm{Hz}$ was undetectable in brains from PcAS-infected mice whereas similar amounts of $\mathrm{Hz}$ were detected in brains of $P b \mathrm{NK} 65$ and PbANKA-infected mice. A possible explanation for this difference is their diverse parasite synchronicity. At the moment of sacrificing the mice and organ removal, the PcAS-parasites in the circulation were all in the ring and young trophozoite stage. As these developmental stages do not yet contain abundant $\mathrm{Hz}[3,10]$, it seemed reasonable that $\mathrm{Hz}$ was not detected in brains from mice infected with this parasite species. It is also possible that no $\mathrm{Hz}$ was detected because PcAS-parasites may not sequester in the brains as is the case for Plasmodium vivax-infected erythrocytes [10]. On the contrary, several developmental stages of $P$. berghei parasites are found in the circulation simultaneously and accumulation of $P$. berghei in the brain is still a debated issue. The observation of similar brain $\mathrm{Hz}$ contents in PbANKA and PbNK65-infected mice cannot be explained by their parasitaemia levels as significantly higher parasitaemias were found in mice that were infected with $P b$ NK65 than in mice infected with PbANKA. The data however do suggest that $\mathrm{Hz}$ as such is not sufficient for the development of this immunopathology as PbNK65infected C57BL/6 J mice do not develop cerebral complications [16]. These data are in contrast with data from Coban et al. [14] and Sullivan et al. [15] who found that brains from mice with cerebral pathology contained more $\mathrm{Hz}$ than healthy brains from infected mice. However, this may be explained by differences in the timing of analysis after infection and in the mouse or parasite strains used in the studies.

Organ-trapped $\mathrm{Hz}$ may originate from two sources. As free $\mathrm{Hz}$ is rapidly removed from the circulation, it is found either inside phagocytes or inside cyto-adhering iRBCs along the endothelial lining of the organs' microvasculature. Systemic perfusion removes circulating iRBCs but not sequestering iRBCs or $\mathrm{Hz}$ inside resident phagocytes, although inadequate perfusion can result from obstruction due to organ-specific cyto-adherence and haemorrhages. Furthermore, it is still not completely clarified if sequestration by murine malaria parasites occurs and which organs are the main targets. Local parasite accumulation has been demonstrated in brains and lungs of PbANKA-infected mice suffering from cerebral symptoms $[18,30]$, but no reports exist on $\mathrm{PbNK} 65$-parasite sequestration. 
After calculating the total amount of $\mathrm{Hz}$ in the mice, it was found that PbANKA and PbNK65-infected mice contained similar amounts of $\mathrm{Hz}$ at comparable parasitaemia levels. This suggests that both parasites produced similar amounts of $\mathrm{Hz}$, or that their schizonts presumably consumed comparable amounts of $\mathrm{Hb}$. On the contrary, lower amounts of $\mathrm{Hz}$ were retrieved in PcASinfected mice despite of similar peripheral parasitaemia. Several explanations can be given for this finding. PcASparasites may produce less $\mathrm{Hz}$, e.g. by digesting less $\mathrm{Hb}$ or by using other haem detoxification mechanisms (transport of haem out of the food vacuole or antioxidative defense mechanisms of the parasite) or PcAS $\mathrm{Hz}$ could be more easily degraded. Interestingly, Noland et al. [31] demonstrated that $\mathrm{Hz}$ crystals from different Plasmodium species have different shapes and dimensions, supporting the notion that $\mathrm{Hz}$ from different species may have different properties. In addition, $\mathrm{Hz}$ contents are variable in RBC infected with different Plasmodium falciparum strains [32].

Another possibility is that peripheral parasitaemia, estimated by counting the percentage of iRBCs by microscopic analysis of Giemsa-stained blood smears, are not a true reflection of the total parasite biomass as they do not take sequestered parasites into account. Consequently, it is possible that PcAS-infected mice contain less $\mathrm{Hz}$ because of lower total parasite burdens. These observations may well translate to the situation in human malaria, where various parasite species have different degrees of virulence. Total parasite biomass in P. falciparum infections is higher than peripheral parasitaemia levels and the difference between these two parameters increases with disease severity [33]. Similarly, $\mathrm{Hz}$-containing peripheral leukocytes are a marker for disease severity [34-36], and accumulation of $\mathrm{Hz}$ in brain micro-vessels is associated with a subtype of cerebral malaria [37]. No data are available yet about total parasite burdens in $P$. vivax-infections and it is still questionable if $P$. vivax-iRBCs can adhere to the endothelial micro-vascular lining. However, cytoadhesion of $P$. vivax-infected erythrocytes was demonstrated in vitro [38] and, despite of the absence of sequestration in the brain [10], it was hypothesized that parasitized RBCs might sequester in lungs from patients with $P$. vivax malaria [39]. Similarly, very little knowledge exists on the role of $\mathrm{Hz}$ in $P$. vivax infections.

Besides differences in pathogenicity, another interesting difference between $P$. berghei and PcAS is that PcAS can be cleared from the circulation in several mouse strains, including C57BL/6 mice, whereas PbANKA and $\mathrm{PbNK} 65$ cannot. The amounts of $\mathrm{Hz}$ produced by these parasites may also contribute to these differences, as $\mathrm{Hz}$ is known to suppress macrophage activity in vitro [40] and in vivo [41]. Interestingly, Spaccapelo et al. found that plasmepsin 4-deficient PbANKA-parasites, which produce less $\mathrm{Hz}$, cause less immunopathology and are more easily cleared by some mouse strains [42].

\section{Conclusions}

This paper describes newly developed and improved methods for sensitive $\mathrm{Hz}$ quantification in mouse organs. Different amounts of $\mathrm{Hz}$ were detected in the analysed organs and total $\mathrm{Hz}$ contents were highest in mice that were infected with lethal parasite strains. Therefore, it is clear that these techniques will be valuable in the investigation of a possible relationship between $\mathrm{Hz}$ and organ-specific malaria pathologies.

\section{Additional file}

\section{Additional file 1: Effect of perfusion on the}

organ-specific haemozoin content. To investigate the effect of perfusion on the organ-specific haemozoin content, mice were infected intraperitoneally with $10^{4} \mathrm{PbNK65}$ parasites. Ten days later, mice were sacrificed, the right lung and kidney were pinched off, and the other organs were perfused with phosphate-buffered saline to remove circulating erythrocytes. The amount of $\mathrm{Hz} / \mathrm{mg}$ tissue was quantified in both perfused and non-perfused lungs and kidneys with the modified 96-well plate based haem-enhanced luminescence assay. The total $\mathrm{Hz}$ content in lung and kidney was calculated by multiplying the amount of $\mathrm{Hz} / \mathrm{mg}$ tissue with the organ weights. As shown in panel A and $\mathrm{B}$, no difference was found in the amount of $\mathrm{Hz}$ in lungs and kidneys with or without perfusion. Nevertheless, perfusion was applied for all organs tested as it is conceptually more rational to remove circulating infected erythrocytes as a source of haemozoin. Each dot represents data of an individual mouse.

\section{Abbreviations}

CM, Cerebral malaria; DB, Densitometric background; DV, Densitometric value; $\mathrm{Hb}$, Haemoglobin; $\mathrm{Hz}$, Haemozoin; MA-ARDS, Malaria-associated acute respiratory distress syndrome; PbANKA, Plasmodium berghei ANKA; PbNK65, Plasmodium berghei NK65; PcAS, Plasmodium chabaudi AS; iRBC, Infected red blood cell; sHz, Synthetic haemozoin (beta-haematin)

\section{Competing interests}

The authors declare that they have no competing interests.

\section{Authors' contributions}

KD participated in the design of the study, performed the animal experiments, optimized the luminescence technique, analysed and interpreted the data, performed the statistical analysis and drafted the manuscript. NL participated in $\mathrm{Hz}$ analysis. SN operated the microscope and designed the script for the densitometric analysis of $\mathrm{Hz}$ on pictures from organ cryosections. EM participated in optimizing the luminescence technique. GO participated in study design, manuscript writing and provided critical support. PVDS conceived and participated in the design of the study, optimized the method for densitometric analysis of $\mathrm{Hz}$ in cryosections and participated in interpreting the data and drafting the manuscript. All authors read and approved the final manuscript.

\section{Acknowledgements}

We want to thank Prof. Jo Van Damme and Prof. Sofie Struyf for the usage of the Axiovert $200 \mathrm{M}$ microscope and Prof. Johan Neyts for the usage of the Precellys homogenizer and the luminometer. Furthermore, we are grateful to Prof. E Schwarzer and Prof. P Arese (University of Turin, Italy) for helpful discussions.

KD is a research assistant of the "Instituut voor Wetenschap en Technologie (IWT)", Belgium. This study was supported by the IWT, the "Fonds voor Wetenschappelijk Onderzoek in Vlaanderen (FWO-Vlaanderen)", the "Geconcerteerde OnderzoeksActies (GOA 2007-2011)", the Rega Centre of Excellence (EF/05/015) and the Research Fund KULeuven (CREA/09/023). 


\section{Author details}

'Laboratory of Immunobiology, Rega Institute, University of Leuven, Leuven, Belgium. 'Laboratory of Molecular Immunology, Rega Institute, University of Leuven, Leuven, Belgium. ${ }^{3}$ Currently at the laboratory of Virology and Chemotherapy, Rega Institute, University of Leuven, Leuven, Belgium.

Received: 17 November 2011 Accepted: 7 March 2012

Published: 14 May 2012

\section{References}

1. Schofield L, Grau GE: Immunological processes in malaria pathogenesis. Nat Rev Immunol 2005, 5:722-735.

2. Goldberg DE: Hemoglobin degradation. Curr Top Microbiol Immunol 2005, 295:275-291.

3. Rosenthal PJ, Meshnick SR: Hemoglobin catabolism and iron utilization by malaria parasites. Mol Biochem Parasitol 1996, 83:131-139.

4. Shio MT, Kassa FA, Bellemare MJ, Olivier M: Innate inflammatory response to the malarial pigment hemozoin. Microbes Infect 2010, 12:889-899.

5. Hanscheid T, Egan TJ, Grobusch MP: Haemozoin: from melatonin pigment to drug target, diagnostic tool, and immune modulator. Lancet Infect Dis 2007, 7:675-685

6. Schwarzer E, Skorokhod OA, Barrera V, Arese P: Hemozoin and the human monocyte-a brief review of their interactions. Parassitologia 2008, 50:143-145.

7. Arese $P$, Schwarzer E: Malarial pigment (haemozoin): a very active 'inert' substance. Ann Trop Med Parasitol 1997, 91:501-516.

8. Grau GE, Mackenzie CD, Carr RA, Redard M, Pizzolato G, Allasia C, Cataldo C, Taylor TE, Molyneux ME: Platelet accumulation in brain microvessels in fatal pediatric cerebral malaria. J Infect Dis 2003, 187:461-466.

9. Dorovini-Zis K, Schmidt K, Huynh H, Fu W, Whitten RO, Milner D, Kamiza S, Molyneux M, Taylor TE: The neuropathology of fatal cerebral malaria in malawian children. Am J Pathol 2011, 178:2146-2158.

10. Silamut K, Phu NH, Whitty C, Turner GD, Louwrier K, Mai NT, Simpson JA, Hien $\Pi$, White $\mathrm{NJ}$ : A quantitative analysis of the microvascular sequestration of malaria parasites in the human brain. Am J Pathol 1999, 155:395-410.

11. Sullivan AD, Nyirenda T, Cullinan T, Taylor T, Lau A, Meshnick SR: Placental haemozoin and malaria in pregnancy. Placenta 2000, 21:417-421.

12. Ismail MR, Ordi J, Menendez C, Ventura PJ, Aponte JJ, Kahigwa E, Hirt R, Cardesa A, Alonso PL: Placental pathology in malaria: a histological, immunohistochemical, and quantitative study. Hum Pathol 2000, 31:85-93.

13. Muehlenbachs A, Fried M, McGready R, Harrington WE, Mutabingwa TK, Nosten F, Duffy PE: A novel histological grading scheme for placental malaria applied in areas of high and low malaria transmission. J Infect Dis 2010, 202:1608-1616

14. Coban C, Ishii KJ, Uematsu S, Arisue N, Sato S, Yamamoto M, Kawai T, Takeuchi O, Hisaeda H, Horii T, Akira S: Pathological role of Toll-like receptor signaling in cerebral malaria. Int Immunol 2007, 19:67-79.

15. Sullivan AD, Ittarat I, Meshnick SR: Patterns of haemozoin accumulation in tissue. Parasitology 1996, 112(Pt 3):285-294.

16. Van den Steen PE, Geurts N, Deroost $K$, Van Al, Verhenne $S$, Heremans $H$ Van Damme J, Opdenakker G: Immunopathology and dexamethasone therapy in a new model for malaria-associated acute respiratory distress syndrome. Am J Respir Crit Care Med 2010, 181:957-968.

17. Craig AG, Grau GE, Janse C, Kazura JW, Milner D, Barnwell JW, Turner G, Langhorne J: The role of animal models for research on severe malaria. PLoS Pathog 2012, 8:e1002401.

18. Haque A, Best SE, Unosson K, Amante FH, de Labastida F, Anstey NM, Karupiah G, Smyth MJ, Heath WR, Engwerda CR: Granzyme B expression by $C D 8+T$ cells is required for the development of experimental cerebral malaria. J Immunol 2011, 186:6148-6156.

19. Mohan A, Sharma SK, Bollineni S: Acute lung injury and acute respiratory distress syndrome in malaria. J Vector Borne Dis 2008, 45:179-193.

20. Valecha N, Pinto RG, Turner GD, Kumar A, Rodrigues S, Dubhashi NG Rodrigues E, Banaulikar SS, Singh R, Dash AP, Baird JK: Histopathology of fatal respiratory distress caused by Plasmodium vivax malaria. Am J Trop Med Hyg 2009, 81:758-762

21. Stephens R, Culleton RL, Lamb TJ: The contribution of Plasmodium chabaudi to our understanding of malaria. Trends Parasitol 2012, 28:73-82.

22. Geurts N, Martens E, Van Aelst I, Proost P, Opdenakker G, Van den Steen PE: Beta-hematin interaction with the hemopexin domain of gelatinase B/MMP-9 provokes autocatalytic processing of the propeptide, thereby priming activation by MMP-3. Biochemistry 2008, 47:2689-2699.
23. Ncokazi KK, Egan TJ: A colorimetric high-throughput beta-hematin inhibition screening assay for use in the search for antimalarial compounds. Anal Biochem 2005, 338:306-319.

24. Schwarzer $E$, Turrini $F$, Arese P: A luminescence method for the quantitative determination of phagocytosis of erythrocytes, of malaria-parasitized erythrocytes and of malarial pigment. Br J Haematol 1994, 88:740-745.

25. Yuan J, Shiller AM: Determination of subnanomolar levels of hydrogen peroxide in seawater by reagent-injection chemiluminescence detection. Anal Chem 1999, 71:1975-1980.

26. Janse CJ, Ramesar J, Waters AP: High-efficiency transfection and drug selection of genetically transformed blood stages of the rodent malaria parasite Plasmodium berghei. Nat Protoc 2006, 1:346-356.

27. http://www.stattools.net/

28. Lawrence C, Olson JA: Birefringent hemozoin identifies malaria. Am J Clin Pathol 1986, 86:360-363.

29. Levesque MA, Sullivan AD, Meshnick SR: Splenic and hepatic hemozoin in mice after malaria parasite clearance. J Parasitol 1999, 85:570-573.

30. Fonager J, Pasini EM, Braks JA, Klop O, Ramesar J, Remarque EJ, Vroegrijk IO, van Duinen SG, Thomas AW, Khan SM, Mann M, Kocken CH, Janse CJ, Franke-Fayard BM: Reduced CD36-dependent tissue sequestration of Plasmodium-infected erythrocytes is detrimental to malaria parasite growth in vivo. J Exp Med 2012, 209:93-107.

31. Noland GS, Briones N, Sullivan DJ Jr: The shape and size of hemozoin crystals distinguishes diverse Plasmodium species. Mol Biochem Parasitol 2003, 130:91-99.

32. Orjih AU, Fitch CD: Hemozoin production by Plasmodium falciparum: variation with strain and exposure to chloroquine. Biochim Biophys Acta 1993, 1157:270-274.

33. Dondorp AM, Desakorn V, Pongtavornpinyo W, Sahassananda D, Silamut K Chotivanich K, Newton PN, Pitisuttithum P, Smithyman AM, White NJ, Day NP: Estimation of the total parasite biomass in acute falciparum malaria from plasma PfHRP2. PLoS Med 2005, 2:e204.

34. Nguyen PH, Day N, Pram TD, Ferguson DJ, White NJ: Intraleucocytic malaria pigment and prognosis in severe malaria. Trans $R$ Soc Trop Med Hyg 1995, 89:200-204.

35. Amodu OK, Adeyemo AA, Olumese PE, Gbadegesin RA: Intraleucocytic malaria pigment and clinical severity of malaria in children. Trans $R$ Soc Trop Med Hyg 1998, 92:54-56.

36. Hanscheid T, Langin M, Lell B, Potschke M, Oyakhirome S, Kremsner PG, Grobusch MP: Full blood count and haemozoin-containing leukocytes in children with malaria: diagnostic value and association with disease severity. Malar J 2008, 7::109.

37. Taylor TE, Fu WJ, Carr RA, Whitten RO, Mueller JS, Fosiko NG, Lewallen S Liomba NG, Molyneux ME: Differentiating the pathologies of cerebral malaria by postmortem parasite counts. Nat Med 2004, 10:143-145.

38. Carvalho BO, Lopes SC, Nogueira PA, Orlandi PP, Bargieri DY, Blanco YC Mamoni R, Leite JA, Rodrigues MM, Soares IS, Oliveira TR, Wunderlich G, Lacerda MV, del Portillo HA, Araujo MO, Russell B, Suwanarusk R, Snounou G, Renia L, Costa FT: On the cytoadhesion of Plasmodium vivax-infected erythrocytes. J Infect Dis 2010, 202:638-647.

39. Anstey NM, Handojo T, Pain MC, Kenangalem E, Tjitra E, Price RN, Maguire GP: Lung injury in vivax malaria: pathophysiological evidence for pulmonary vascular sequestration and posttreatment alveolar-capillary inflammation. J Infect Dis 2007, 195:589-596.

40. Schwarzer E, Turrini F, Ulliers D, Giribaldi G, Ginsburg H, Arese P: Impairment of macrophage functions after ingestion of Plasmodium falciparum-infected erythrocytes or isolated malarial pigment. J Exp Med 1992, 176:1033-1041

41. Scorza T, Magez S, Brys L, De Baetselier P: Hemozoin is a key factor in the induction of malaria-associated immunosuppression. Parasite Immuno 1999, 21:545-554

42. Spaccapelo R, Janse CJ, Caterbi S, Franke-Fayard B, Bonilla JA, Syphard LM, Di Cristina M, Dottorini T, Savarino A, Cassone A, Bistoni F, Waters AP, Dame $\mathrm{JB}$, Crisanti A: Plasmepsin 4-deficient Plasmodium berghei are virulence attenuated and induce protective immunity against experimental malaria. Am J Pathol 2010, 176:205-217.

doi:10.1186/1475-2875-11-166

Cite this article as: Deroost et al: Improved methods for haemozoin quantification in tissues yield organ-and parasite-specific information in malaria-infected mice. Malaria Journal 2012 11:166. 